\title{
Bursting behavior of the Galactic center faint X-ray transient GRS 1741.9-2853
}

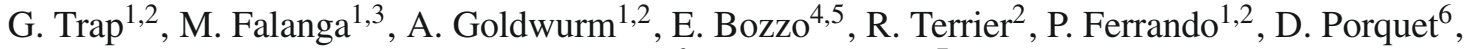 \\ N. Grosso 6 , and M. Sakano ${ }^{7}$
}

1 Service d'Astrophysique (SAp) / IRFU / DSM / CEA Saclay, Bât. 709, 91191 Gif-sur-Yvette Cedex, France e-mail: trap@apc.univ-paris7.fr

2 AstroParticule \& Cosmologie (APC) / Université Paris VII / CNRS / CEA / Observatoire de Paris, Bât. Condorcet, 10 rue Alice Domon et Léonie Duquet, 75205 Paris Cedex 13, France

3 International Space Science Institute (ISSI), Hallerstrasse 6, 3012 Bern, Switzerland

${ }^{4}$ Istituto Nazionale Di Astrofisica (INAF) / Osservatorio Astronomico di Roma, via Frascati 33, Monte Porzio Catone, 00044 Rome, Italy

5 INTEGRAL Science Data Center (ISDC) / Science data center for Astrophysics, Ch. d'Ecogia 16, 1290 Versoix (Ge), Switzerland

6 Observatoire astronomique de Strasbourg / Université de Strasbourg / CNRS / INSU, 11 rue de 1'Université, 67000 Strasbourg, France

7 Department of Physics and Astronomy / University of Leicestern, LE1 7RH Leicester, UK

Received 3 February 2009 / Accepted 11 June 2009

\section{ABSTRACT}

\begin{abstract}
Aims. The neutron star low-mass X-ray binary GRS 1741.9-2853 is a known type-I burster at the Galactic center. It is transient, faint, and located in a very crowded region, only $10^{\prime}$ from the supermassive black hole Sgr A ${ }^{\star}$. Therefore, its bursting behavior has been poorly studied so far. In particular, its persistent emission has rarely been detected between consecutive bursts, because of lack of sensitivity or confusion. This caused GRS 1741.9-2853 to be one of the nine "burst-only sources" identified by BeppoSAX a few years ago. The physical properties of GRS 1741.9-2853 bursts are of significant interest beacause we know little about the nuclear regimes of low accretion rate bursters. For the first time we analyze several bursts along with the persistent emission of the source, using INTEGRAL, XMM-Newton, and Swift observations.

Methods. We investigate the source flux variability and bursting behavior during its 2005 and 2007 long outbursts. These events were monitored almost entirely by INTEGRAL, both in the soft (3-20 keV) and hard (20-100 keV) X-ray bands, and also partly by XMM-Newton and Swift (2-10 keV) in 2007.

Results. In its last activity periods, 2005 and 2007, the persistent luminosity of GRS 1741.9-2853 varied between $\sim 1.7$ and $10.5 \times$ $10^{36} \mathrm{erg} \mathrm{s}^{-1}$, i.e., $0.9-5.3 \%$ of the Eddington luminosity. The shape of the spectrum as described by an absorbed power-law remained with a photon index $\Gamma \approx 2$ and a column density $N_{\mathrm{H}} \approx 12 \times 10^{22} \mathrm{~cm}^{-2}$ throughout the outbursts. We discovered 11 type-I bursts with INTEGRAL, and inspected four additional bursts: two recorded by XMM-Newton and two by Swift. From the brightest burst, we derive an upper limit to the source distance of $\sim 7 \mathrm{kpc}$. The observed bursts characteristics and source accretion rate infer pure helium explosions igniting at column depths $y_{\mathrm{ign}} \approx 0.8-4.8 \times 10^{8} \mathrm{~g} \mathrm{~cm}^{-2}$, for typical energy releases of $\sim 1.2-7.4 \times 10^{39} \mathrm{erg}$.
\end{abstract}

Key words. Galaxy: center - stars: neutron - X-rays: individuals: GRS 1741.9-2853 - X-rays: binaries - X-rays: bursts

\section{Introduction}

A low-mass X-ray binary (LMXB) is a stellar system consisting of a low mass star $\left(<1 M_{\odot}\right)$ filling its Roche lobe and pouring matter onto a compact object via an accretion disk. A large fraction $(\sim 55 \%)$ of LMXBs in our Galaxy are transient (see Liu et al. 2007, for a catalogue), which means they display bright $\mathrm{X}$-ray outbursts from time to time, lasting from weeks to months. During such an event, they typically increase their luminosity by a factor of at least a hundred compared to the dim state in which they spend most of their time and are usually too faint to be detected. One traditionally distinguishes three classes of transients according to their $2-10 \mathrm{keV}$ peak luminosities: bright transients $\left(10^{37-38} \mathrm{erg} \mathrm{s}^{-1}\right)$, faint transients $\left(10^{36-37} \mathrm{erg} \mathrm{s}^{-1}\right)$, and very faint transients $\left(10^{34-36} \mathrm{erg} \mathrm{s}^{-1}\right)$ (e.g., Wijnands et al. 2006). In particular, faint transients were recognized as a distinctive class with the observations of BeppoSAX (Heise et al. 1999). Just as for other transients, the outbursts of the faint ones can be accomodated by the classical disk instability model (King \& Ritter 1998; Lasota 2001, for a review), provided their orbital periods are between 80-120 min (King 2000). Yet, these faint transients are markedly concentrated towards the Galactic center (GC) (in't Zand 2001), and generally harbor a neutron star as a compact object, which is inferred from the presence of type-I X-ray bursts in the light curves of these systems. Type-I bursts are thought to result from thermonuclear explosions on the solid surface of the neutron star (see Lewin et al. 1993; Strohmayer \& Bildsten 2006 for reviews, and Cornelisse et al. (2003); Galloway et al. (2008); Chelovekov et al. (2007) for catalogues). Interestingly, the properties of the bursts are tightly bound to the accretion rate of the neutron star. Early theoretical work by Fujimoto et al. (1981) showed that, at low accretion rates, the bursts should be triggered by the unstable burning of accreted hydrogen and should thus be dissimilar to the usual "pure helium" bursts encountered at higher accretion rates. From an observational stand point, the study of low accretion 
rate bursters developed with the discovery of nine "burst-only sources" by BeppoSAX (Cocchi et al. 2001; Cornelisse et al. 2004). The persistent emission - and so the accretion rate of these objects was so low that the source could never be detected right before and after the bursts. This subclass of sources has also motivated theoretical studies suggesting that, at the lowest accretion rates, unstable $\mathrm{H}$ burning triggers vigorous $\mathrm{He}$ flashes in a H-rich environment, whereas at slightly higher acretion rates, weak pure $\mathrm{H}$ flashes build up a thick He layer underneath the surface (Peng et al. 2007; Cooper \& Narayan 2007). This layer can eventually ignite, leading to a several minute long burst. Dim bursters could therefore possibly help link short bursts and the increasing number of intermediate long bursts (Falanga et al. 2008, 2009, and references therein). We also emphasize that almost all currently known X-ray recycled millisecond pulsars are faint LMXB transients (Wijnands 2006; Falanga 2008), making these sources interesting targets in which to look for fast rotators.

In this context, we present the observational analysis of one of the "burst-only sources", GRS 1741.9-2853, with all the data pertaining to the GC ever collected by INTEGRAL and XMMNewton since February 2002, and Swift data from early 2007.

\subsection{History of GRS 1741.9-2853 from 1990 to 2004}

GRS 1741.9-2853 was discovered in the GC in spring 1990 by ART-P, the low energy instrument onboard the Granat satellite (Sunyaev 1990; Sunyaev et al. 1991). The source was seen at a constant flux level of $2 \times 10^{-10} \mathrm{erg} \mathrm{s}^{-1} \mathrm{~cm}^{-2}$ (4-30 keV) during two observations in March and April, and was also present in the mosaics of Granat high energy instrument SIGMA (40-100 keV, Goldwurm 1996). However in all subsequent campaigns on the GC, Granat failed to detect GRS 1741.9-2853, establishing the source as a transient. We note that this binary is only $10^{\prime}$ from the Milky Way supermassive black hole Sgr A ${ }^{\star}$, and was thus in the field of view (FOV) of numerous observations carried out by high energy satellites (see below). In September of 1994, ASCA identified GRS 1741.9-2853 (a.k.a. AX J1745.0-2855) in outburst with a flux of $1.8 \times 10^{-12} \mathrm{erg} \mathrm{s}^{-1} \mathrm{~cm}^{-2}$ in the $0.7-10 \mathrm{keV}$ band (Sakano et al. 2002).

Two years later (August and September 1996), BeppoSAX recorded three type-I X-ray bursts from GRS 1741.92853 with its Wide Field Camera (Cocchi et al. 1999), implying that the source is a LMXB housing an accreting neutron star. From a photospheric radius expansion (PRE) episode in the brightest burst, Cocchi et al. (1999) derived a distance of $\sim 8 \mathrm{kpc}$ for GRS 1741.9-2853, placing it close to the very center of the Galaxy. This was based on the assumption that, during PRE, the bolometric peak luminosity of a burst saturates at the Eddington luminosity and thus acts as a standard candle (Lewin et al. 1993; Kuulkers et al. 2003). We note that BeppoSAX could not measure the persistent flux of the source and set an upper limit to the bolometric luminosity of $\sim 1.6 \times 10^{36} \mathrm{erg} \mathrm{s}^{-1}$, which made this burster another member of the "burst-only sources" class (Cornelisse et al. 2004). A little earlier in 1996, from April to July, RXTE scanned the GC and detected eight bright bursts from an unidentified source in this intricate region. Even though the FOV of RXTE/PCA contained many bursters, Galloway et al. (2008) attributed the bursts to GRS 1741.9-2853 in light of the activity seen by BeppoSAX at roughly the same time and the similarity between the bursts caught by the two satellites. Interestingly, three of the RXTE bursts showed burst oscillations at a frequency of $589 \mathrm{~Hz}$ (Strohmayer et al. 1997), so that if the association of the source and the bursts holds, the neutron star in GRS 1741.9-2853 is spinning with a period of only
Table 1. GRS 1741.9-2853 flux history.

\begin{tabular}{lccc}
\hline \hline Date & Instrument & $\begin{array}{c}\text { Flux }^{a} \\
{\left[10^{-11} \mathrm{erg} \mathrm{s}^{-1} \mathrm{~cm}^{-2}\right]}\end{array}$ & Ref. $^{b}$ \\
\hline 1990-2002 & $\ldots \ldots \ldots \ldots \ldots \ldots$ & {$[1]$} \\
2002 Feb. 26 & XMM-Newton/PN & $<0.0007$ & {$[2]$} \\
2002 Oct. 3 & XMM-Newton/PN & 1 & {$[2]$} \\
2004 Mar. 28 & XMM-Newton/PN & $<0.0007$ & {$[2]$} \\
2004 Aug. 31 & XMM-Newton/PN & $<0.0002$ & {$[2]$} \\
2005 Apr. 10 & INTEGRAL/ISGRI & 31.9 & {$[3],[4]$} \\
2005 Jun. 5 & Chandra/HRC & 10 & {$[5],[6]$} \\
2005 Jul. 1 & Chandra/ACIS & 1.7 & {$[5],[6]$} \\
2006 Feb. 27 & XMM-Newton/PN & $<0.0058$ & {$[2]$} \\
2006 Sep. 8 & XMM-Newton/PN & $<0.0057$ & {$[2]$} \\
2006 Sep. 20 & Swift/XRT & 1.2 & {$[7]$} \\
2007 Feb. 15 & INTEGRAL/ISGRI & 13 & {$[4],[8]$} \\
2007 Feb. 16 & Swift/XRT & 15 & {$[9],[2]$} \\
2007 Feb. 22 & Chandra/ACIS & 3 & {$[10]$} \\
2007 Feb. 27 & XMM-Newton/PN & 11.6 & {$[2]$} \\
2007 Mar. & Swift/XRT & 26 & {$[7]$} \\
2007 Apr. 2 & XMM-Newton/PN & 13.1 & {$[11],[2]$} \\
2007 Apr. 4 & XMM-Newton/PN & 5.5 & {$[11],[2]$} \\
2007 Sep. 6 & XMM-Newton/PN & $<0.0026$ & {$[2]$} \\
2008 Mar. 23 & XMM-Newton/PN & $<0.0003$ & {$[2]$} \\
\hline
\end{tabular}

${ }^{a} \mathrm{X}$-ray flux in the ranges $2-8 \mathrm{keV}$ for XMM-Newton and Chandra, 20-60 keV for INTEGRAL/ISGRI, 3-10 keV for INTEGRAL/JEM-X, and $2-10 \mathrm{keV}$ for Swift/XRT; ${ }^{b}$ references: [1] Muno et al. (2003), [2] This work, [3] Kuulkers et al. (2007c), [4] Kuulkers et al. (2007b), [5] Wijnands et al. (2005), [6] Wijnands et al. (2006), [7] Degenaar \& Wijnands (2009), [8] Kuulkers et al. (2007a), [9] Wijnands et al. (2007), [10] Muno et al. (2007), [11] Porquet et al. (2007).

$1.7 \mathrm{~ms}$. Because of confusion, the PCA could not measure the persistent flux of the source. Unlike RXTE, ASCA managed to detect this persistent emission from GRS 1741.9-2853 in September 1996 (Sakano et al. 2002), and so strengthened the association of the RXTE with BeppoSAX bursts into a long outburst of the source that lasted at least six months. Thanks to good statistics, physical spectral parameters were constrained. The persistent emission X-ray spectrum was fitted with a photoelectrically absorbed power-law model, whose parameters are the column density, $N_{\mathrm{H}}=11.4_{-0.8}^{+0.9} \times 10^{22} \mathrm{~cm}^{-2}$, and the spectral photon index, $\Gamma=2.36 \pm 0.16$. The average flux was $1 \times 10^{-10} \mathrm{erg} \mathrm{s}^{-1} \mathrm{~cm}^{-2}(0.7-10 \mathrm{keV})$.

The binary was then undetected for four years, until Chandra witnessed the source in outburst in fall 2000, with an associated weak thermonuclear burst (Muno et al. 2003) ${ }^{1}$. These authors fitted the spectrum of the persistent emission with an absorbed power-law, $N_{\mathrm{H}}=(9.7 \pm 0.2) \times 10^{22} \mathrm{~cm}^{-2}$ and $\Gamma=1.88 \pm 0.04$. They also found the source to be in quiescence in summer 2001, at a luminosity of $\sim 10^{32} \mathrm{erg} \mathrm{s}^{-1}$ (2$8 \mathrm{keV}$ ). These Chandra measurements provided the most accurate position of GRS 1741.9-2853 to date: $\alpha=17^{\mathrm{h}} 45^{\mathrm{m}} 2.33^{\mathrm{s}}, \delta=$ $-28^{\circ} 54^{\prime} 49.7^{\prime \prime}$ (J2000), with an uncertainty of $0.7^{\prime \prime}$. By browsing the XMM-Newton archive, we found that in October 2002, GRS 1741.9-2853 was again weakly active at a luminosity of $\sim 10^{35} \mathrm{erg} \mathrm{s}^{-1}(2-8 \mathrm{keV})$ (see Sect. 3.2.1), which is reminescent of the weak outburst captured by $A S C A$ in 1994. XMMNewton upper limits to the burster flux until 2004 are given in Table 1.

\footnotetext{
1 GRS 1741.9-2853 is also known as CXOGC J174502.3-285450 in the Chandra catalogue (Muno et al. 2006).
} 


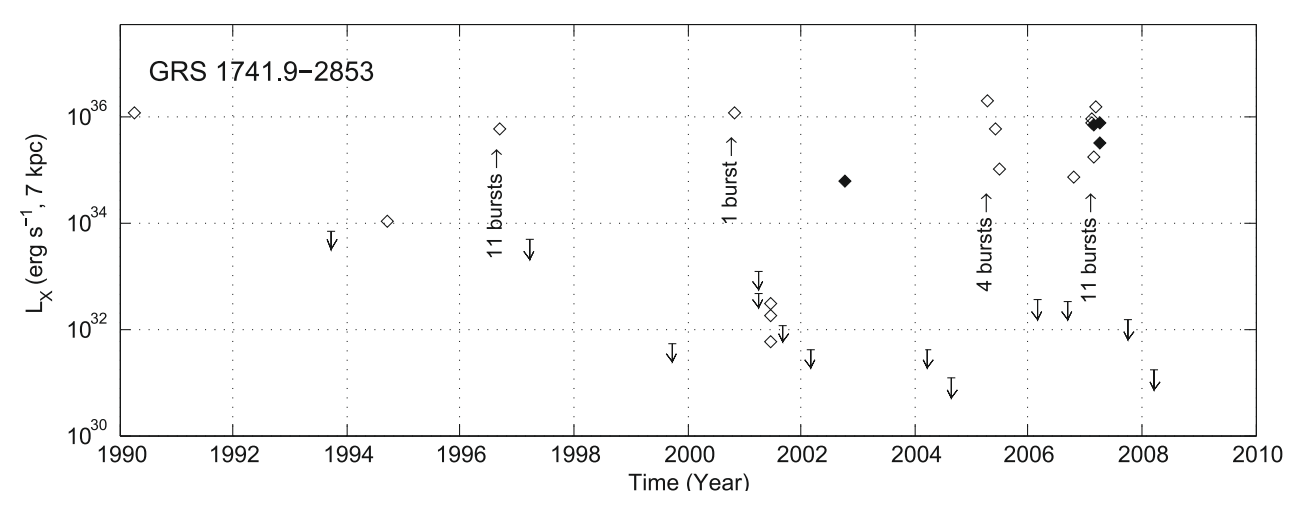

Fig. 1. X-ray luminosity history of GRS 1741.9-2853. Open diamonds and upper limits before 2002 correspond to data published elsewhere, and filled diamonds to the XMM-Newton measurements reported here. See Table 1 for the respective energy ranges of the different points. We indicate the total number of bursts detected during each outburst by BeppoSAX, RXTE, Chandra, INTEGRAL, Swift, and XMM-Newton.

\subsection{The 2005 and 2007 outbursts}

From February to April 2005, the hard X-ray imager INTEGRAL/IBIS/ISGRI perceived a new outburst from GRS 1741.9-2853 (Kuulkers et al. 2007c). The source was temporally called IGR J17453-2853 until it was later recognized as GRS 1741.9-2853 (Kuulkers et al. 2007b). Chandra found the source still in activity in June (Wijnands et al. 2005) and slowly fading one month later (Wijnands et al. 2006). The July spectrum of the source was similar to the previous Chandra fit published earlier by Muno et al. (2003): $N_{\mathrm{H}}=10.5_{-3.7}^{+4.9} \times 10^{22} \mathrm{~cm}^{-2}$ and $\Gamma=1.8_{-0.8}^{+1}$. These observations triggered an optical follow-up in the $I$ band, which could not identify any visible counterpart (Laycock et al. 2005), in accordance with the high reddening expected from GC sources. As we explain below (Sect. 3.1.2), during this long outburst of at least three months, we found four type-I X-ray bursts with INTEGRAL/JEM-X.

In 2007, renewed activity from GRS 1741.9-2853 was detected by several observatories. In February, INTEGRAL started a new long exposure of the Galactic bulge, and clearly caught the source in activity (Kuulkers et al. 2007a,b). The satellite monitored the outburst all the way to April when the source went back to quiescence. We discovered a total of seven bursts distributed between March and April (see Sect. 3.1.2). The early detection of the source by INTEGRAL triggered a short pointing with Swift/XRT, which detected a new X-ray burst (Wijnands et al. 2007). It is likely that another burst of GRS 1741.92853 was also detected by Swift/BAT earlier, in January (Fox et al. 2007). These two bursts are also inspected in Sect. 3.3.2 of the present paper. From March to April, Swift/XRT followed the source more regularly, and measured the peak of the outburst at a luminosity of $\sim 2 \times 10^{36} \mathrm{erg} \mathrm{s}^{-1}(2-10 \mathrm{keV}$ ) (Degenaar $\&$ Wijnands 2009). The latter authors fitted the average spectrum with $N_{\mathrm{H}}=14_{-0.9}^{+1} \times 10^{22} \mathrm{~cm}^{-2}$ and $\Gamma=2.6 \pm 0.2$. They also noticed the presence of a short (one week long) and faint $\left(\sim 10^{35} \mathrm{erg} \mathrm{s}^{-1}\right)$ outburst of the source in September 2006, which might have been a precursor to the 2007 longer outburst. Chandra measured the persistent luminosity of GRS $1741.9-2853$ on February $22^{\text {nd }}$ to be $4 \times 10^{35} \mathrm{erg} \mathrm{s}^{-1}$ (described by a model with parameters $N_{\mathrm{H}}=9 \pm 0.5 \times 10^{22} \mathrm{~cm}^{-2}$ and $\Gamma=0.9 \pm 0.1,2-8 \mathrm{keV}$ ) (Muno et al. 2007). On February 27th, an XMM-Newton/PN observation was carried out in Timing mode to search for millisecond pulsations in the persistent emission and burst oscillations. Yet, a burst was neither recorded nor was any pulsation found (Wijnands 2008). Finally, XMM-Newton was pointed once again to the GC at the beginning of April as part of a multiwavelength campaign on the supermassive black hole Sgr A* (Porquet et al. 2008; Trap et al. 2009; Dodds-Eden et al. 2009). This long observation ( 100 ks) coincided with the end of the GRS 1741.9-2853 outburst and contains two type-I bursts (Porquet et al. 2007); we examine these in greater details in Sect. 3.2.2.

To summarize the flux history of GRS 1741.9-2853, we updated in Fig. 1 the long term light curves extracted from Muno et al. (2003) and Wijnands et al. (2006), which represents data spanning from 2002 to 2007 (Table 1). Over the past 18 years, the X-ray luminosity of GRS 1741.9-2853 has varied regularly from $10^{32} \mathrm{erg} \mathrm{s}^{-1}$ in quiescence, up to $10^{36} \mathrm{erg} \mathrm{s}^{-1}$ in outburst, justifying its source classification as a faint soft X-ray transient.

\section{Observations and data analysis}

\subsection{INTEGRAL}

The INTEGRAL satellite (Winkler et al. 2003) has been regularly scanning the GC twice a year, in spring and fall, since the beginning of the mission in October 2002. Hereafter, we take into account the data of the Joint European X-ray Monitor (JEM-X), module 1 and 2 (Lund et al. 2003), between 3 and $20 \mathrm{keV}$, and the INTEGRAL Soft Gamma-Ray Imager (ISGRI, 20-100 keV) (Lebrun et al. 2003), from the imager onboard the INTEGRAL satellite (IBIS) (Ubertini et al. 2003). The data were processed with the standard offline science analysis (OSA) software package, version 7.0, distributed by the INTEGRAL science data center (Courvoisier et al. 2003) and based on algorithms described in Goldwurm et al. (2003) for IBIS, and Westergaard et al. (2003) for JEM-X.

To search for periods of activity of GRS 1741.9-2853 with JEM-X, we selected all the public data since the launch of the satellite, pointing less than $3.5^{\circ}$ away from the source position. The available data consist of 2626 usable individual pointings. These single pointings were first deconvolved and analyzed separately. We then divided the dataset into six-month periods and combined the corresponding individual pointings into mosaics. GRS 1741.9-2853 was clearly detected only during two periods, from February to April 2005 and February to April 2007, at a significance level of $21 \sigma$ and $12 \sigma$, respectively. Figure 2 (upper panel) shows the significance map of the GC region, in the 3-20 keV energy range, for the 2007 dataset. Many sources are present in the FOV of all the mosaics, but because of the to JEM-X high angular resolution (3.3' FWHM at best, Brandt et al. 2003), GRS 1741.9-2853 is free of contamination from the neighboring objects. Thereafter, we concentrate on these two 
Table 2. XMM-Newton/PN and Swift/XRT log of the observations used in this paper.

\begin{tabular}{lcccc}
\hline \hline Orbit & ObsID & $\begin{array}{c}\text { Date Start } \\
{[\mathrm{UTC}]}\end{array}$ & $\begin{array}{c}\text { Exposure } \\
{[\mathrm{ks}]}\end{array}$ & Mode $^{2}$ \\
\hline \multicolumn{5}{c}{ XMM-Newton/PN } \\
406 & 111350101 & 2002 Feb. 26, 06:41:42 & 40.0 & Imaging (T) \\
516 & 111350301 & 2002 Oct. 3, 07: 17:07 & 15.3 & Imaging (T) \\
788 & 202670501 & 2004 Mar. 28, 16:45:33 & 41.9 & Imaging (M) \\
866 & 202670701 & 2004 Aug. 31, 03:34:59 & 126.7 & Imaging (M) \\
1139 & 302882601 & 2006 Feb. 27, 04:27:37 & 0.5 & Imaging (M) \\
1236 & 302884001 & 2006 Sep. 8, 17:19:46 & 0.5 & Imaging (M) \\
1322 & 506291201 & 2007 Feb. 27, 06:27:39 & 37.6 & Timing (M) \\
1338 & 402430701 & 2007 Mar. 30, 21:28:14 & 32.3 & Imaging (M) \\
1339 & 402430301 & 2007 Apr. 1, 15:07:59 & 103.5 & Imaging (M) \\
1340 & 402430401 & 2007 Apr. 3, 16:39:17 & 97.6 & Imaging (M) \\
1418 & 504940201 & 2007 Sep. 6, 10:28:34 & 11.1 & Imaging (M) \\
1518 & 505670101 & 2008 Mar. 23, 17:22:10 & 96.6 & Imaging (M) \\
\hline \multicolumn{5}{c}{ Swift/XRT } \\
$\ldots$ & 0003088801 & 2007 Feb 16, 21:39:18 & 3.9 & Photon counting \\
\hline
\end{tabular}

epochs, 2005 and 2007, which gather 417 and 253 individual pointings of $\sim 1.8 \mathrm{ks}$ each, for total effective exposures of about $397 \mathrm{ks}$ and $242 \mathrm{ks}$, respectively.

\subsection{XMM-Newton}

GRS 1741.9-2853 has was detected on three occasions by the XMM-Newton satellite (Jansen et al. 2001) operating in different intrument configurations (see Table 2 for a journal of the observations): October 2002, February 2007, and March/April 2007. The science analysis software (SAS, version 7.1.2) and the latest current calibration files (CCFs) were used to produce calibrated event lists from the observation data files (ODFs). PN event lists were then filtered with the standard selection criteria $\mathrm{Flag}=0$ to remove bad pixels, hot pixels, and pixels close to CCD gaps.

For all the observations, we computed the light curve of the entire detector in the $10-12 \mathrm{keV}$ energy range to identify the time intervals contaminated by background soft proton flares. We excluded from our analysis all the phases where this background count rate exceeded 100 counts per time bins of $100 \mathrm{~s}$. In particular, the last seven hours of both rev-1339 and rev-1340 were rejected in this context. Apart from rev-1322, where the standard technique for timing data was applied, all spectra from the persistent emission were extracted from a circular region of 50 " radius, encircling $90 \%$ of the point spread function (PSF) of the instrument. Background counts were obtained from a similar region offset from the source position. Because the bursts periods were affected by pile-up, special care was taken for the light curves and spectra of these time intervals: events from the inner 6 " of the PSF were ignored and the parameter Pattern was set to zero to reject all but single events. For each burst, we took the persistent emission before the burst as background. Version 11.3.2. of the XSPEC software (Arnaud 1996) was used to fit all the spectra with physical models. Errors are quoted at a $90 \%$ confidence level for one parameter of interest.

Concerning the observations where GRS 1741.92853 was not active, i.e., February 2002, March/August 2004, February/September 2006, September 2007, and March 2008 , we calculated $3 \sigma$ upper limits in counts $\mathrm{s}^{-1}$ with ximage and converted them into $2-8 \mathrm{keV}$ unabsorbed fluxes by using WebPIMMS. Results are summarized in Table 1.

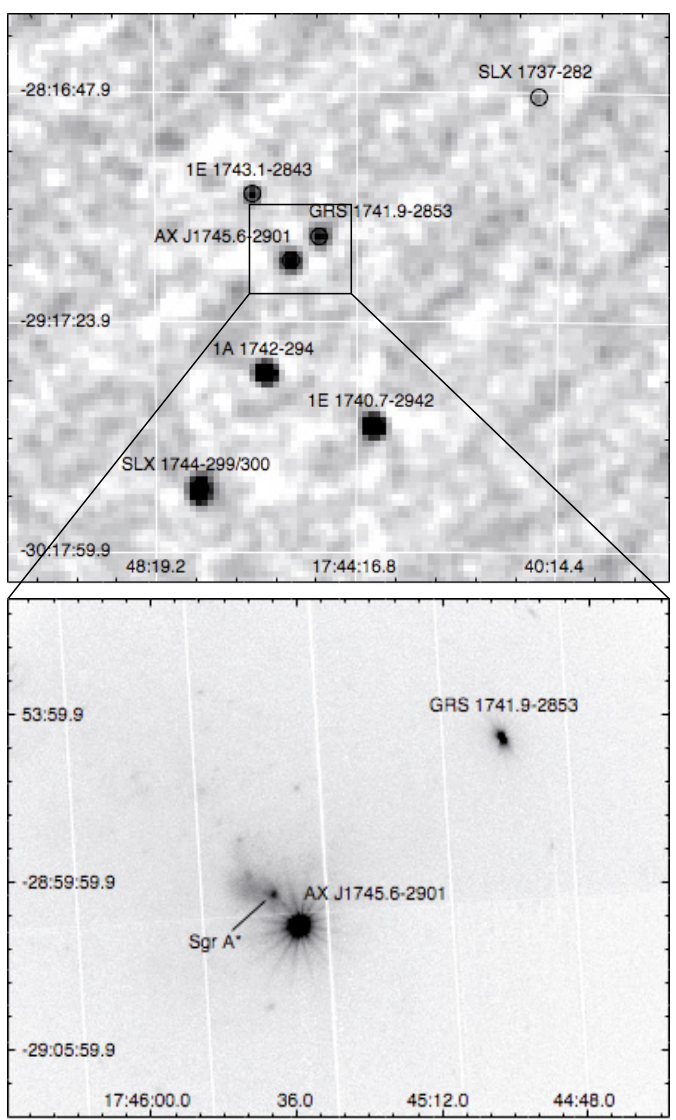

Fig. 2. INTEGRAL/JEM-X 3-20 keV significance mosaic (top pannel) constructed from 253 individual pointings between February and April 2007, with an effective exposure of $242 \mathrm{ks}$ at the position of GRS 1741.9-2853. Equatorial coordinates are used and the Galactic plane runs from upper left to bottom right. The magnified view (lower panel) is an XMM-Newton/PN 0.1-10 keV image in April 2007 (rev$1340,97.6 \mathrm{ks}$ exposure). Sgr $\mathrm{A}^{\star}$, the supermassive black hole of the Galactic center, is clearly apparent since this observation contains several flares from the vicinity of the black hole, enhancing its average luminosity (Porquet et al. 2008).

\subsection{Swift}

For the present study we used the publicly accessible observations id. 00257213000 and 00030888001 from Swift (Gehrels et al. 2004). The first dataset includes the burst discovered on January 22nd, 2007 with the high energy coded mask detector BAT (Fox et al. 2007), whereas the second comprises both the burst and the persistent emission observed with the low energy telescope XRT on February 16-17th, 2007 (Wijnands et al. 2007). After the burst detected by the Swift/BAT on January 22nd, no XRT follow-up has been performed. The total exposure time was $\sim 4 \mathrm{ks}$ for BAT during observation 00257213000 , and $\sim 3.9$ ks for XRT during observation 00030888001.

For the BAT data analysis, we used the batgrbproduct tool included in the Heasoft package 6.6.1. A BAT spectrum was extracted for the entire duration of the burst $(9 \mathrm{~s})$. The XRT data were processed with the xrtpipeline (version 0.12.1) task. Filtering and screening criteria were applied by using the ftools (Heasoft version 6.6.1). We analyzed data in photon counting (PC) mode, and selected event grades of 0-12. All the XRT/PC data were affected by a strong pile-up, and thus we extracted 
the source light curves and spectra by using annular regions centered on the source. The persistent light curves and spectra were extracted through an annular region with an inner radius of 4 pixels and an external radius of 20 pixels. Unfortunately, the count rate during the burst was very high and the corresponding pile-up was much stronger than that of the persistent emission. To correct for this effect, we used annular regions with different inner radii during the rise, the peak, and the decay of the burst (the inner radii were 10,15, and 4 pixels, respectively). We then used the xrtlccorr task to account for these corrections in the background-subtracted light curves. To estimate the spectral properties of the source and its flux during the entire burst, we used an extraction region with an inner radius of 10 pixels. All the spectra were rebinned to ensure that there was at least 20 photons per bin permit $\chi^{2}$ fitting. Exposure maps were created by means of the xrtexpomap routine, and we used the latest spectral redistribution matrices in the HEASARC calibration database (version 0.1.1). Ancillary response files, accounting for different extraction regions, vignetting and PSF corrections, were generated using the xrtmkarf task.

\section{Results}

\subsection{INTEGRAL}

\subsubsection{Outbursts}

Albeit significant in the global mosaics of the 2005 and 2007 outbursts, the source was so weak that it was detected significantly in neither the JEM-X nor ISGRI individual pointings. Hence, the standard technique for deriving light curves with OSA was inadequate. So, we therefore compiled the light curves from the individual images, by collecting the count rate in the pixel consistent with the source position for ISGRI, and by integrating the flux over a $4.2^{\prime}(F W H M)$ PSF for JEM$\mathrm{X}^{3}$. By rebinning the data into long intervals ( $\sim$ days), the evolution in the outbursts becomes visible (see Fig. 3). In 2005, INTEGRAL observations ceased in April, but Chandra still detected the source fading in June and July (Wijnands et al. 2005, 2006) so INTEGRAL missed the end of the ouburst. In 2007, instead, we missed the beginning and but observed the end. We note that the drop in the light curve around MJD 54155 (end of April 2007) was confirmed by independent flux measurements of Swift, Chandra, and XMM-Newton at lower energies in the 2-10 keV band (see Table 1).

\subsubsection{Type-I bursts}

To search for type-I bursts, we investigated all the JEM-X, module 1 and 2, data publicly available since the beginning of the mission, pointing less than $3.5^{\circ}$ away from the source position, and forced the standard pipeline to construct $2 \mathrm{~s}$ rebinned light curves. Since the persistent emission of GRS 1741.9-2853 was never detected in single pointings, these light curves contain mainly background emission and "spikes". Most of these spikes are due to instrumental artefacts and some to contamination by bright type-I bursts from other sources in the FOV. To identify the burst origins, we systematically compiled images of the sky between the beginning and the end of the burst candidates. When a thermonuclear burst from GRS 1741.9-2853 occurs, one should locate a significant excess at the position of the source in the image. By these means, we found a total of between four

3 The size of the PSF was empirically derived from a bright source of the FOV, GX 3+1, in the $3-20 \mathrm{keV}$ range.
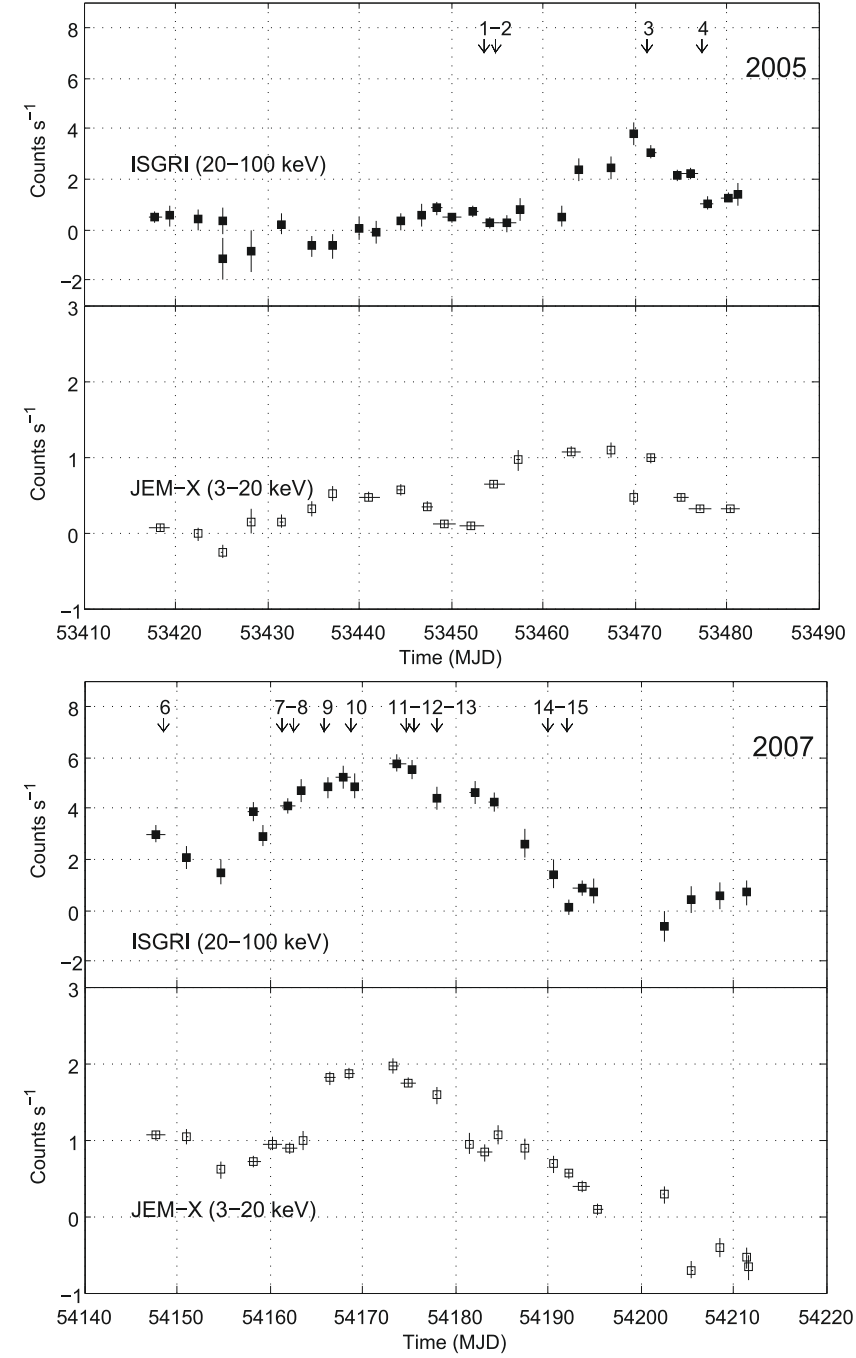

Fig. 3. INTEGRAL/ISGRI and JEM-X light curves of the 2005 (upper panel) and 2007 (lower panel) outbursts in the $20-100 \mathrm{keV}$ and 3-20 keV bands, respectively. All the type-I bursts reported in this work are indicated by the arrows, leaving out the $S$ wift/BAT burst \#5 that occured on $\sim$ MJD 54122. Note that the error bars on the count rate and the binning times are sometime within the square symbols. Negative count rates are due to statistical fluctuations at low flux level after background subtraction.

and seven type-I bursts from GRS 1741.9-2853 in the 2005 and 2007 outbursts, respectively, and none during the quiescent state of the source.

We also looked for the high energy (18-40 keV) tails of these 11 bursts in the ISGRI data. Therefore, we extracted the off-axis corrected light curves with events selected according to the detector illumination pattern, using a pixel illumination fraction threshold of 0.4 . Only the brightest burst, \#4, could be convincingly detected in the light curve and image. In so far as the FOV from IBIS is larger than the one of JEM-X, the total IBIS exposure on GRS 1741.9-2853 is longer and so it was worth searching for additional bursts in the IBIS data stream. To do that, we used the INTEGRAL burst alert system (IBAS) software running at ISDC and dedicated to the real-time localization and discovery of gamma-ray bursts, transient X-ray sources, and bursts in the IBIS/ISGRI data (Mereghetti et al. 2003). IBAS identified only burst \#4.

In Table 4, we report the burst start times measured by JEM$\mathrm{X}$. The start time of each burst is defined as the time when the 
intensity rose to $10 \%$ of the peak above the persistent intensity level. In Fig. 4, we plot the background subtracted light curves of the 11 bursts with a $2 \mathrm{~s}$ time bin. The background subtraction consisted in removing the constant level present before and after the bursts in the light curve of the individual pointing of interest. We note that this background was not caused by the persistent emission of the source, because it was too weak to be detected in such a short period $(\sim 1.8 \mathrm{~s})$. The rise time is the interval between the start of the burst and the time at which the intensity reaches $90 \%$ of the peak intensity. For all the bursts, it was $\sim 2-4$ s, except maybe for burst 4 , which had an unusual morphology. The efolding decay times, determined for the time interval after the peaks and plateaus, are specified in Table 4 . They range roughly between 10 and $20 \mathrm{~s}$. The total durations, i.e., from the burst start time back to the persistent flux level in the $3-20 \mathrm{keV}$ band, were around 20-30 s.

For the spectral analysis, we used JEM-X counts during $5 \mathrm{~s}$ at the peak of the bursts, in the $3-20 \mathrm{keV}$ band. The net peak burst spectra are well fitted by a photoelectrically absorbed blackbody model (BB). Unfortunately, the energy range covered by JEM-X does not allow us to constrain the interstellar hydrogen column density, $N_{\mathrm{H}}$. We therefore fixed $N_{\mathrm{H}}$ to $12 \times 10^{22} \mathrm{~cm}^{-2}$, the value found with XMM-Newton (see Sect. 3.2.1), in all our spectral fits. The inferred BB temperatures, $k T_{\text {peak }}$, and apparent BB radii, $R_{\text {peak }}$, at $7 \mathrm{kpc}$ (see Sect. 4) are listed in Table 4, along with other bursts parameters. The peak fluxes, $F_{\text {peak }}$, were derived from the 3-20 keV light curves peak count rates with $2 \mathrm{~s}$ time resolution and renormalized for the $0.1-100 \mathrm{keV}$ bolometric energy range. The bursts fluences are obtained from the fluxes extrapolated in the bolometric energy band over the respective burst durations.

To investigate the bursting behavior of a LMXB, it is important to know its accretion rate before the bursts (see Sect. 4). A common estimator of this parameter is the persistent luminosity of the source. Here, to assess the persistent flux before one burst, we first compiled JEM-X mosaics consisting of between 10 and 30 consecutive pointings preceding the burst in a single band (3$20 \mathrm{keV}$ ). Except for bursts 1 and 2 where only upper limits could be set, the persistent emission of the source was always detected significantly in the mosaics. We then converted the count rate fitted in the mosaics into a bolometric flux ${ }^{4}$, assuming one constant spectral shape throughout the outbursts, that consisted of an intrinsic power-law of index $\Gamma=2$ between 0.1 and $100 \mathrm{keV}$ (see Sect. 3.2.1).

\subsection{XMM-Newton}

\subsubsection{Persistent emission}

The EPIC/PN spectra of GRS 1741.9-2853 persistent emission in 2002 and 2007 were fitted most accurately by an absorbed power-law. They were fitted between $2-8 \mathrm{keV}$ since, outside these bounds, there were systematic residuals due to the background. Table 3 presents these best-fit results. We studied all the spectra with $N_{\mathrm{H}}$ as a free parameter. Given that we do not measure any variation in $N_{\mathrm{H}}$, the observed absorption is most likely mainly caused by the Galactic absorption and not instrinsic to the source. So, we refitted all the data consistently with $N_{\mathrm{H}}$ fixed to $11.9 \times 10^{22} \mathrm{~cm}^{-2}$, the value with the smallest error found with the February 2007 pointing. In 2007, the index remained always close to 2, thus justifying the assumption we made in Sect. 3.1.2.

4 This count rate was compatible with the rebinned light curves on Fig. 3 but more precise.

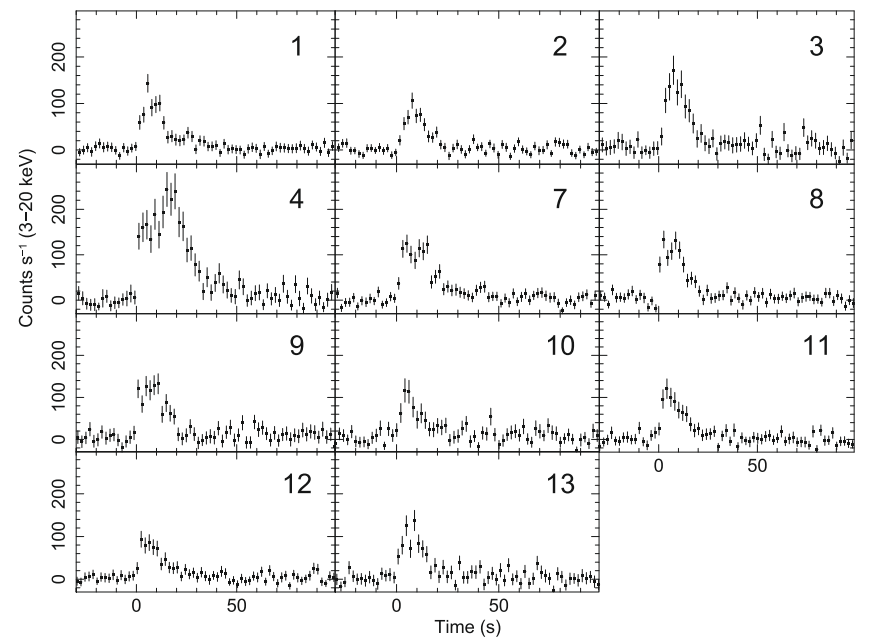

Fig. 4. Background subtracted light curves of all the bursts detected by INTEGRAL/JEM-X in the 3-20 keV energy range. The time bin is $2 \mathrm{~s}$ and the start times of the bursts are given in Table 4.

Table 3. XMM-Newton/PN and Swift/XRT spectral results for the persistent emission.

\begin{tabular}{|c|c|c|c|c|}
\hline Date $^{a}$ & $\begin{array}{c}N_{\mathrm{H}}^{b} \\
{\left[10^{22} \mathrm{~cm}^{-2}\right]}\end{array}$ & $\Gamma$ & $F_{\text {pers }}{ }^{c}$ & $\chi^{2} /$ d.o.f. \\
\hline \multirow{3}{*}{2002 Oct. 3} & & $\overline{M-N e w t o n / 1}$ & & \\
\hline & $14 \pm 3$ & $2.0 \pm 0.5$ & $1.0 \pm 0.2$ & $67 / 64$ \\
\hline & [11.9] & $1.6 \pm 0.2$ & $0.9 \pm 0.2$ & $68 / 65$ \\
\hline 2007 Feb. 27 & $11.9 \pm 0.2$ & $1.83 \pm 0.03$ & $11.60 \pm 0.05$ & $219 / 197$ \\
\hline \multirow[t]{2}{*}{2007 Mar. 30} & $11.6 \pm 0.4$ & $1.76 \pm 0.08$ & $11.9 \pm 0.1$ & $155 / 200$ \\
\hline & [11.9] & $1.81 \pm 0.04$ & $12.2 \pm 0.1$ & $156 / 201$ \\
\hline \multirow[t]{2}{*}{2007 Mar. 31} & $12.1 \pm 0.4$ & $1.86 \pm 0.08$ & $12.0 \pm 0.1$ & $192 / 210$ \\
\hline & [11.9] & $1.83 \pm 0.04$ & $11.9 \pm 0.1$ & $192 / 211$ \\
\hline \multirow[t]{2}{*}{2007 Apr. 1} & $12.0 \pm 0.3$ & $2.08 \pm 0.05$ & $13.6 \pm 0.1$ & $242 / 213$ \\
\hline & [11.9] & $2.06 \pm 0.02$ & $13.5 \pm 0.1$ & $242 / 214$ \\
\hline \multirow[t]{2}{*}{2007 Apr. 2} & $12.5 \pm 0.3$ & $2.14 \pm 0.05$ & $13.1 \pm 0.1$ & $213 / 195$ \\
\hline & [11.9] & $2.04 \pm 0.02$ & $12.4 \pm 0.1$ & $225 / 196$ \\
\hline \multirow[t]{2}{*}{2007 Apr. 3-4 } & $13.1 \pm 0.4$ & $2.30 \pm 0.08$ & $5.5 \pm 0.1$ & $179 / 200$ \\
\hline & [11.9] & $2.08 \pm 0.03$ & $4.9 \pm 0.1$ & $204 / 201$ \\
\hline 2007 Feb. 16-17 & $10.9 \pm 3.6$ & $\begin{array}{c}\text { Swift/XRT } \\
1.9 \pm 0.7\end{array}$ & $19.0 \pm 3.0$ & $31 / 28$ \\
\hline
\end{tabular}

${ }^{a}$ For 2007, Mar. 30 and 31 designate the periods just before and after burst 12 respectively (see Fig. 5). Same thing for Apr. 1 and 2 and burst 13. Apr. 3-4 period is the last XMM-Newton revolution (rev$1340) ;{ }^{b}$ whenever there are brackets, the column density is frozen; ${ }^{c}$ unabsorbed flux in the $2-8 \mathrm{keV}$ and $2-10 \mathrm{keV}$ band in units of $10^{-11}$ $\mathrm{erg} \mathrm{s}^{-1} \mathrm{~cm}^{-2}$ for XMM-Newton/PN and Swift/XRT, respectively.

for the persistent emission measured by JEM-X. The flux was divided by two for the last orbit. For this observation, we created an image cleaned for out of time events (OoT) in the $0.1-10 \mathrm{keV}$, displayed in Fig. 2. The light curve of the three last observations in March/April 2007 are plotted in Fig. 5.

\subsubsection{Type-I bursts}

In the light curve of the three last revolutions (Fig. 5), we clearly recognize a couple of type-I X-ray bursts (14 and 15), separated by $\sim 178 \mathrm{ks}$. In view of the huge data gap between them, we cannot claim that the bursts were consecutive. A closer view of their net light curves (from which the persistent emission was subtracted) with a time bin of $2 \mathrm{~s}$ is visible in Fig. 6 (top pannels). Both bursts have quick rises ( $2 \mathrm{~s}$ ) followed by exponential decays of about $20 \mathrm{~s}$ (see Table 4 ).

The statistics obtained with XMM-Newton allowed us to perform time-resolved spectral analysis of the bursts. Again, the 


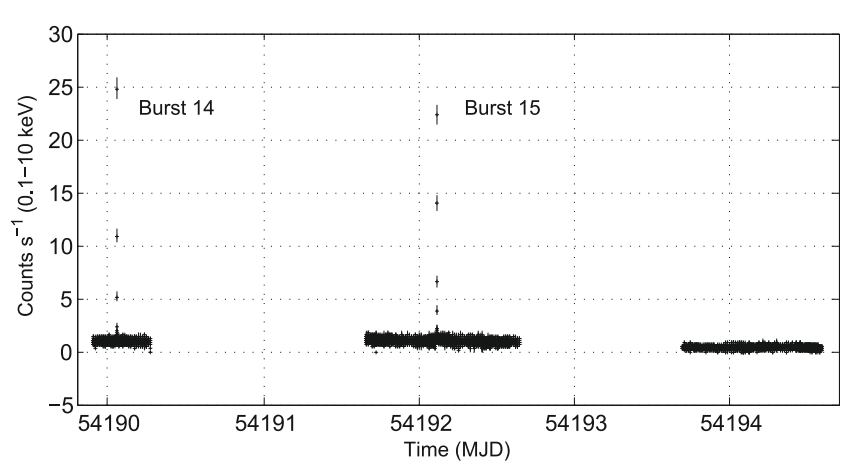

Fig. 5. XMM-Newton/PN background subtracted light curve of GRS 1741.9-2853 in spring 2007 (rev-1338, 1339, and 1340). Two type-I bursts, 14 and 15 , are clearly visible.

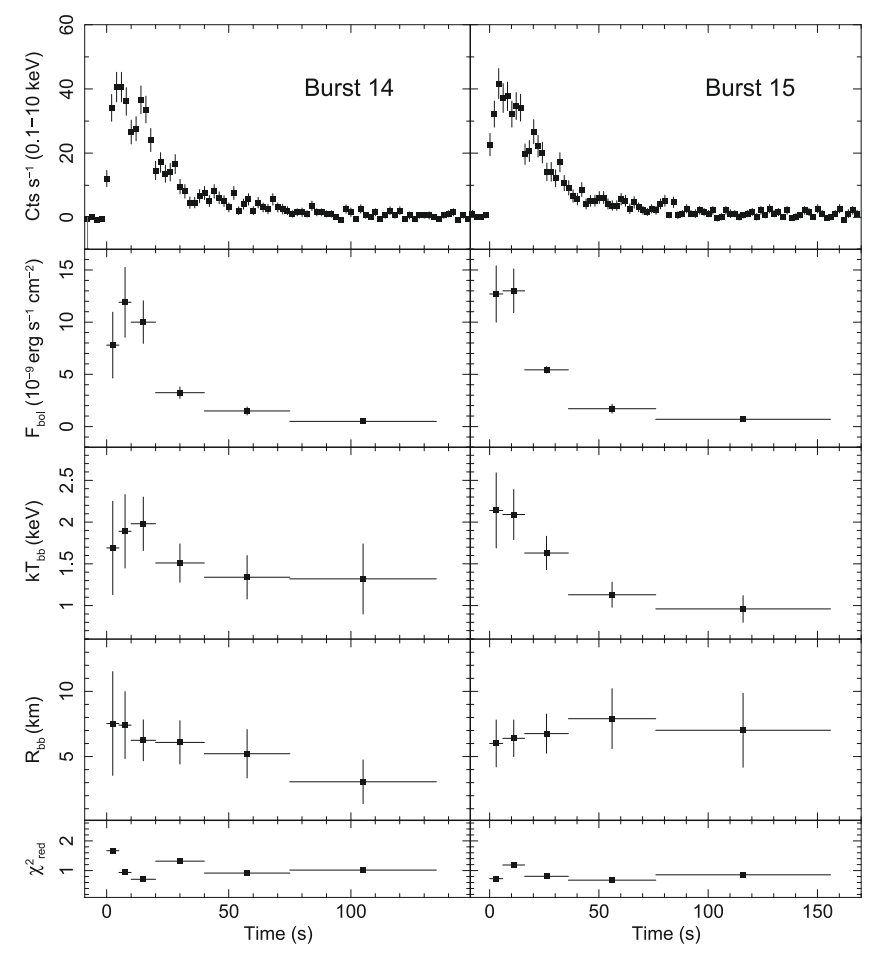

Fig. 6. Light curves and spectral evolution of bursts 14 and 15 as measured by XMM-Newton/PN in spring 2007. From top to bottom: count rate with a time bin of $2 \mathrm{~s}$, bolometric flux estimated over $0.1-100 \mathrm{keV}$, temperature and radius of the photosphere obtained by fitting the spectra with the BB model, and reduced chi-squared of the fits.

spectra were well fitted by absorbed blackbodies with $N_{\mathrm{H}}$ fixed to $12 \times 10^{22} \mathrm{~cm}^{-2}$. Figure 6 shows the evolution in the bolometric flux $(0.1-100 \mathrm{keV}), F_{\mathrm{bol}}$, blackbody temperature, $k T_{\mathrm{bb}}$, and blackbody radius, $R_{\mathrm{bb}}$, as a function of time. In Table 4 , we report these latter values measured at the peak, as well as the fluence, $f_{\mathrm{b}}$, integrated over the total duration of the bursts. The first event, $\# 14$, was not contemporaneous with the INTEGRAL GC survey, whereas \#15 occured during an INTEGRAL observing window. Nonetheless we detected it neither with ISGRI nor JEM-X. The ISGRI non-detection is consistent with the burst being faint, while for JEM-X the absence of detection is surely due to the position of the source at the noisy edge of the FOV.

To determine the persistent flux prior to the bursts, we extrapolated the results indicated in Table 3 over the $0.1-100 \mathrm{keV}$ energy band.

\subsection{Swift}

\subsubsection{Persistent emission}

The Swift/XRT persistent emission spectrum of 2007 February 16-17 was fitted by an absorbed power-law model, as used for the XMM-Newton spectra. The best-fit model parameters are $N_{\mathrm{H}}=10.9 \pm 3.6 \times 10^{22} \mathrm{~cm}^{-2}$ with a power-law photon index of $\Gamma=1.9 \pm 0.7$ with a $\chi_{\text {red }}^{2}=31.5 / 28$. The averaged absorbed and unabsorbed $2-10 \mathrm{keV}$ fluxes were $9.8 \pm 2.5 \times$ $10^{-11} \mathrm{erg} \mathrm{s}^{-1} \mathrm{~cm}^{-2}$ and $19.0 \pm 0.7 \times 10^{-11} \mathrm{erg} \mathrm{s}^{-1} \mathrm{~cm}^{-2}$, respectively. These spectral parameters are in accordance with the previously reported values (Wijnands et al. 2007). We note that this observation was contemporaneous with the beginning of the INTEGRAL/JEM-X/ISGRI 2007 observations. The XRT unabsorbed bolometric flux $0.1-100 \mathrm{keV}, \sim 8.1 \times 10^{-10} \mathrm{erg} \mathrm{s}^{-1} \mathrm{~cm}^{-2}$, is comparable with the first pre-burst JEM-X flux reported in Table 4 for burst 7, which occurred at the same flux level (see light curve in Fig. 3 bottom panels). For a long-term XRT light curve of GRS 1741.9-2853, we refer the reader to Fig. 2 in Degenaar \& Wijnands (2009).

\subsubsection{Type-I bursts}

Swift detected two type-I bursts: one, \#5, with BAT (15-25 keV) on 2007 January 22 at 06:12:54 (UTC) and another one, \#6, with XRT, 25.69976 days later. For the BAT burst, we cannot determine the cooling time, $\tau$, since only the hard tail of the burst was observed. However, the rise time was around $2 \mathrm{~s}$ and the total burst elapsed time was $8.2 \mathrm{~s}$. Because of the short exposure time, the persistent emission could not be measured, in addition, no low energy Swift/XRT follow-up was performed to investigate the persistent emission. The BAT and XRT light curve properties are reported in Table 4 . We note that the other burst also found at high energy was burst number 4 with INTEGRAL/ISGRI. In Fig. 7, we show the Swift/BAT and Swift/XRT bursts and, for comparison with BAT, the 15-25 keV ISGRI burst. The BAT burst was not covered by the INTEGRAL scan of the GC and the XRT burst unluckily occurred during a slew of INTEGRAL, preventing the use of the data.

The spectral analysis of burst \#5 was carried out in the 15$25 \mathrm{keV}$. The spectrum was well fitted by an absorbed blackbody model with the column density being again frozen to $12 \times 10^{22} \mathrm{~cm}^{-2}$. The inferred bursts parameters are reported in Table 4 . The measured unabsorbed flux was extrapolated to the 0.1-100 keV band by generating dummy responses with XSPEC. This extrapolation was well justified for the JEM-X data since the blackbody temperature was well inside the instrument bandpass (Sect. 3.1.2). However, this is not the case for the BAT burst,

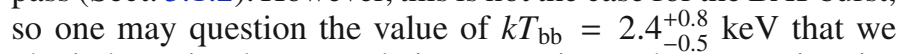
obtain by a simple extrapolation. To estimate the uncertainty introduced by this method, we focused on burst \#4 and compared the fit of the ISGRI data alone with the joint JEM-X/ISGRI spectrum $(3-35 \mathrm{keV})$. We find that fitting solely the high energy spectrum and extrapolating it to softer energies, leads to an error of $30 \%$, which is inside our BAT error box and so is an acceptable method.

Due to the high count rate at the peak of burst \#6, the XRT/PC image was affected by strong pile-up and a spectral analysis of the burst peak was impossible. Therefore, to estimate the burst peak flux, we used the parameters determined for the burst mean spectrum $\left(N_{\mathrm{H}}\right.$ fixed at $12 \times 10^{22} \mathrm{~cm}^{-2}$, and BB temperature $1.6_{-0.3}^{+0.4} \mathrm{keV}$ ) and calculated the flux at the peak by using the burst peak count rate $\left(96 \pm 30\right.$ counts $\left.^{-1}\right)$ and the 

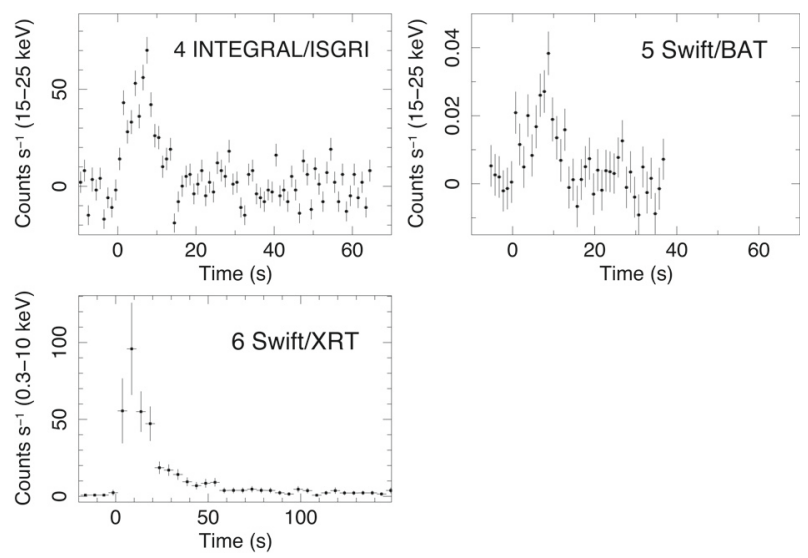

Fig. 7. Background subtracted light curves of the Swift bursts and the ISGRI one for comparison with the BAT one. The time bins are $1 \mathrm{~s}$ for $\# 4$ and \#5, and $5 \mathrm{~s}$ for \#6. The start times are given in Table 4.

online tool webpimms. We found an unabsorbed $0.1-100 \mathrm{keV}$ flux of $2.7 \pm 0.8 \times 10^{-8} \mathrm{erg} \mathrm{s}^{-1} \mathrm{~cm}^{-2}$. The burst peak BB temperature could however be higher than the used $1.6 \mathrm{keV}$. Therefore, using webpimms we also estimated the peak flux for a BB temperature of $3 \mathrm{keV}$. Since this is believed to be an upper limit to the X-ray temperature at the burst peak, the corresponding X-ray flux we derived $\left(6 \times 10^{-8} \mathrm{erg} \mathrm{s}^{-1} \mathrm{~cm}^{-2}\right)$ could reasonably be considered to be an upper limit to the peak X-ray flux.

\section{Discussion}

Herein, we have reported the monitoring by INTEGRAL and XMM-Newton of the Galactic center faint transient GRS 1741.92853 in outburst during two visibility periods, 2005 and 2007. During these outbursts, the bolometric persistent flux of the source was observed to vary between $\sim 0.3$ and $1.8 \times$ $10^{-9} \mathrm{erg} \mathrm{s}^{-1} \mathrm{~cm}^{-2}$, a spectral photon index always beaing close to 2 (Tables 3 and 4). We discovered 11 type-I bursts with INTEGRAL and also examined four other ones: two via XMMNewton and two via Swift, to be as complete as possible. The parameters of the eight $R X T E$ bursts reported by Galloway et al. (2008) are very similar, i.e., peak fluxes, bursts fluences, rise times, and shapes, to those summarized in Table 4. This argues in favor of the association proposed by these authors between the bursts and the source.

The distance to the source can be determined by bursts undergoing PRE. We cannot claim that any of the bursts detected for GRS 1741.9-2853 reached a PRE, but from the brightest one, \#4, we can calculate an upper limit to the source distance. Assuming an isotropic emission and a bolometric peak luminosity equal to the Eddington value for a pure He type-I burst (hydrogen mass fraction $X=0), L_{\mathrm{Edd}}=3.8 \times 10^{38} \mathrm{erg} \mathrm{s}^{-1}$, as empirically derived by Kuulkers et al. (2003), we obtain a distance upper limit $d=7.6_{-0.5}^{+2.0} \mathrm{kpc}$. For comparison, the theoretical value (Lewin et al. 1993) for the upper limit found by considering a $\mathrm{He}$ atmosphere and a canonical neutron star $\left(1.4 M_{\odot}\right.$ for $10 \mathrm{~km}$ radius) is $6.7_{-0.4}^{+1.8} \mathrm{kpc}$. We note also that taking a hydrogen-rich mixed burst (solar composition $X=0.7$ ) would lead to a closer distance upper limit of $5.1_{-0.3}^{+1.4} \mathrm{kpc}$. These results agree with the distance estimates of Cocchi et al. (1999) and Galloway et al. (2008), and the measured high column density $\sim 12 \times 10^{22} \mathrm{~cm}^{-2}$.

In the following, we assume that the bursts we observed were pure He bursts $(X=0)$ and the source distance has a fiducial value $d=7 \mathrm{kpc}$. The unabsorbed bolometric persistent flux range of the source then translates to luminosities
$L_{\text {pers }} \approx 1.7-10.5 \times 10^{36} \mathrm{erg} \mathrm{s}^{-1}$. This persistent luminosity depends on the local accretion rate per unit area, $\dot{m}$, according to the equation $L_{\text {pers }}=4 \pi R^{2} \dot{m}(G M / R)(1+z)^{-1}$, where $M$ and $R$ represent the mass and radius of the neutron star, respectively, and $z=\left(1-2 G M /\left(R c^{2}\right)\right)^{-1 / 2}-1$ is the gravitational redshift at its surface. We consider here the canonical values $M=1.4 M_{\odot}, R=10 \mathrm{~km}$, and consequently $z=0.31$. As a result, $\dot{m} \approx 1000-6100 \mathrm{~g} \mathrm{~s}^{-1} \mathrm{~cm}^{-2}$. A common unit for expressing $\dot{m}$ is the corresponding Eddington rate $\dot{m}_{\mathrm{Edd}}=2 m_{\mathrm{p}} c\left(R \sigma_{\mathrm{Th}}\right)^{-1}(1+$ $\left.X_{0}\right)^{-1}(1+z)$, where $m_{\mathrm{p}}, X_{0}, c$, and $\sigma_{\text {Th }}$ are the proton mass, the $\mathrm{H}$ abundance of the matter accreted from the donor star, the speed of light, and the Thompson scattering cross section, respectively. From now on, we assume that $X_{0}=0.7$ and so $\dot{m}_{\text {Edd }}=11.5 \times 10^{4} \mathrm{~g} \mathrm{~s}^{-1} \mathrm{~cm}^{-2}$, leading to $\dot{m} \approx 0.9-5.3 \% \dot{m}_{\text {Edd }}$.

On the other hand, the observed fluences, $f_{\mathrm{b}}$, of the bursts listed in Table 4, infer total energies radiated during the bursts of $E_{\mathrm{b}}=4 \pi d^{2} f_{\mathrm{b}} \approx 1.2-7.4 \times 10^{39} \mathrm{erg}$. We can thereby estimate the ignition column of each burst, $y_{\text {ign }}$, using the relation $y_{\text {ign }}=$ $E_{\mathrm{b}}(1+z) /\left(4 \pi R^{2} \epsilon_{\text {nuc }}\right)$, where $\epsilon_{\text {nuc }}$ is the nuclear energy released per unit mass that can be related to the nuclear energy released per nucleon, $Q_{\text {nuc }} \approx 1.6+4 X \mathrm{MeV}_{\text {nucleon }}{ }^{-1}$, by $\epsilon_{\text {nuc }}=Q_{\text {nuc }} \times$ $10^{18} \mathrm{erg} \mathrm{g}^{-1}$ (Wallace \& Woosley 1981; Fujimoto et al. 1987). This yields ignition columns of $y_{\text {ign }} \approx 0.8-4.8 \times 10^{8} \mathrm{~g} \mathrm{~cm}^{-2}$.

Theoretical recurrence times for the bursts, $\tau_{\text {rec }}=$ $\left(y_{\text {ign }} / \dot{m}\right)(1+z)$, can then be evaluated to be $\approx 0.2-43.9 \times 10^{5} \mathrm{~s}=$ $0.2-5.1$ days. We emphasize that this derivation of $\tau_{\text {rec }}$ is independent of the assumed distance $d$. The observed times, $\Delta t$, between bursts (Table 4) and these expected recurrence times for pure He combustion, $\tau_{\text {rec }}$, agree well with the data for bursts 3 and 4 , and therefore suggest they were consecutive. The same conclusion holds for bursts 1 and 2, presuming that the persistent flux in-between was close to $0.3 \times 10^{-9} \mathrm{erg} \mathrm{s}^{-1} \mathrm{~cm}^{-2}$, although we could not detect it. To place stronger constraints on the recurrence time, we consider the longest uninterrupted data set, i.e., the three consecutive XMM-Newton revolutions of April 2007. Since the theoretical recurrence time is nearly twice the measured $\Delta t$ between bursts 14 and 15 , we certainly missed one burst during the data gap in the light curve shown in Fig. 5. In contrast, by repeating the previous calculations with a higher hydrogen abundance in the nuclear burning, $X=0.7$, we infer shorter recurrence times. In particular, for the XMM-Newton bursts, $\tau_{\text {rec }}$ drops to 0.3 days, which means that there should have been two bursts surrounding burst \#15 in the light curve of revolution 1339 in Fig. 5. Since we did not observe these bursts, we conclude that GRS 1741.9-2853 displays pure He bursts.

Theoretical works now predict that the nature of the nuclear burning of the accreted material $(\mathrm{H}$ and/or He) during a type-I burst depends critically on the accretion rate of the neutron star (Fujimoto et al. 1981; Bildsten 1998; Peng et al. 2007; Cooper \& Narayan 2007). We consider three independent contiguous mass ranges. If $0 \lesssim \dot{m} / \dot{m}_{\mathrm{Edd}} \lesssim 0.01$ (i), $\mathrm{H}$ burns then unstably by means of the cold $\mathrm{CNO}$ cycle, and so ignites a mixed $\mathrm{He} / \mathrm{H}$ explosion. Since the fusion of $\mathrm{H}$ involves slow $\beta$-decays, the rise time and total duration of the burst should be relatively long: $\sim 10 \mathrm{~s}$ and $\sim 100 \mathrm{~s}$, respectively. For $0.01 \lesssim \dot{m} / \dot{m}_{\text {Edd }} \lesssim 0.1$ (ii), $\mathrm{H}$ is burned into He steadily through the hot CNO cycle, and a pure He layer develops underneath the surface, which may ignite by means of the unstable $3 \alpha$ process, thereby producing a pure He burst. The strong interaction at work implies a shorter rise time and total duration $(\sim 1 \mathrm{~s}$ and $\sim 10 \mathrm{~s}$, respectively). Finally, if $0.1 \lesssim \dot{m} / \dot{m}_{\text {Edd }} \lesssim 1$ (iii), $\mathrm{H}$ is not burned stably fast enough so that a pure He flash triggers a mixed $\mathrm{He} / \mathrm{H}$ burst with short rise time $(\sim 1 \mathrm{~s})$ and long duration $(\sim 100 \mathrm{~s})$. Here, the persistent 
Table 4. Burst parameters.

\begin{tabular}{|c|c|c|c|c|c|c|c|c|c|c|c|c|}
\hline Id. & & $\begin{array}{c}\text { Burst Start Time } \\
\text { [UTC] }\end{array}$ & $\begin{array}{c}k T_{\text {peak }}^{b} \\
{[\mathrm{keV}]}\end{array}$ & $\begin{array}{c}R_{\text {peak }} c \\
{[\mathrm{~km}]}\end{array}$ & $\chi^{2} /$ d.o.f. & $F_{\text {peak }}{ }^{d}$ & $f_{\mathrm{b}}{ }^{e}$ & $\begin{array}{c}\tau_{\mathrm{fit}^{f}} \\
{[\mathrm{~s}]}\end{array}$ & $\begin{array}{c}\tau_{\text {calc }^{g}} \\
{[\mathrm{~s}]}\end{array}$ & $F_{\text {pers }}{ }^{h}$ & $\begin{array}{l}\Delta t^{i} \\
{[\mathrm{~s}]}\end{array}$ & $\begin{array}{c}\gamma^{j} \\
{\left[10^{-3}\right]}\end{array}$ \\
\hline 1 & JEM-X & 2005 Mar. $24,14: 26: 23$ & $2.3_{-0.5}^{+0.8}$ & $7_{-3}^{+5}$ & $15.7 / 12$ & $3.2_{-15}^{+0.7}$ & $3.6 \pm 0.4$ & $12_{-2}^{+2}$ & $11 \pm 3$ & $<0.3$ & & $<9$ \\
\hline 2 & JEM-X & 2005 Mar $25,22: 21: 43$ & $2.0_{-04}^{+0.5}$ & $8_{-3}^{+5}$ & $8.7 / 9$ & $2.8_{-16}^{+0.6}$ & $2.6 \pm 0.3$ & $8_{-1}^{+2}$ & $9 \pm 2$ & $<0.3$ & 114869 & $<11$ \\
\hline 3 & JEM-X & 2005 Apr. $11,08: 53: 16$ & $1.9_{-0.5}^{+0.4}$ & $12_{-12}^{+12}$ & $13.7 / 17$ & $4.2_{-34}^{+1.0}$ & $4.5 \pm 0.5$ & $8_{-1}^{+\frac{1}{2}}$ & $11 \pm 3$ & $0.7 \pm 0.2$ & 1420269 & $17 \pm 7$ \\
\hline 4 & JEM-X* & 2005 Apr. 17, 06:18:54 & $2.5_{-0 .}^{-0.5}$ & $\begin{array}{c}12-12 \\
8_{-2}^{+3}\end{array}$ & $18.4 / 22$ & $\begin{array}{l}5.5_{-3}^{+0.4} \\
-1.7\end{array}$ & $12.6 \pm 1.0$ & $\begin{array}{l}-1 \\
11_{-2}^{+2}\end{array}$ & $23 \pm 3$ & $0.4 \pm 0.2$ & 509114 & $7.7 \pm 4$ \\
\hline 5 & BAT & 2007 Jan. $22,06: 12: 54$ & $2.4^{-0.4}$ & $8^{-5}$ & $5.2 / 7$ & $5.8^{-1.1}$ & $>4.7$ & & $>8$ & & & ? \\
\hline 6 & XRT & 2007 Feb. 16, 22:59:28 & & & & $\begin{array}{l}2.7_{-0.8}^{+0.5} \\
+0.8\end{array}$ & $3.3 \pm 0.6$ & $13_{-5}^{+6}$ & $12 \pm 6$ & $0.8 \pm 0.1$ & 2220459 & $29 \pm 12$ \\
\hline 7 & JEM-X & 2007 Mar 02, 08:42:34 & $1.6_{-03}^{+0.3}$ & $15_{-5}^{+8}$ & $9.1 / 13$ & $3.3_{-15}^{+0.8}$ & $5.3 \pm 0.5$ & $16_{-3}^{+2}$ & $16 \pm 3$ & $0.7 \pm 0.2$ & 1158185 & $20 \pm 6$ \\
\hline 8 & JEM-X & 2007 Mar 03, 15:51:05 & $1.9_{-0.3}^{+0.3}$ & $11_{-3}^{+4}$ & $11.6 / 12$ & $3.5_{-1 .}^{-1.5}$ & $3.9 \pm 0.4$ & $11_{-2}^{+1}$ & $11 \pm 2$ & $1.0 \pm 0.2$ & 112057 & $29 \pm 7$ \\
\hline & JEM-X & 2007 Mar. 06, 22:35:16 & $1.6_{-03}^{-0.3}$ & $16_{-7}^{+13}$ & $9.1 / 11$ & $3.6_{-2.3}^{-1.2 .6}$ & $4.3 \pm 0.5$ & $10_{-2}^{+3}$ & $12 \pm 2$ & $1.3 \pm 0.2$ & 283423 & $36 \pm 8$ \\
\hline & JEM-X & 2007 Mar. 09, 17:28:56 & $2.8_{-10}^{+3.3}$ & $4_{-4}^{+1}$ & $5.9 / 9$ & $2.8_{-25}^{+1.2}$ & $2.5 \pm 0.5$ & $12_{-2}^{+-4}$ & $9 \pm 4$ & $1.2 \pm 0.2$ & 240790 & $43 \pm 20$ \\
\hline & JEM-X & 2007 Mar. 15, 16:01:05 & $1.7^{-1.05}$ & $14^{-4}$ & $8.1 / 10$ & $3.1_{-2.5}^{+0.8}$ & $2.5 \pm 0.4$ & $11_{-2}^{+2}$ & $8 \pm 3$ & $1.8 \pm 0.2$ & 513094 & $58 \pm 16$ \\
\hline & JEM-X & 2007 Mar 16, 14:09:14 & $2.2^{-1.0}$ & $\begin{array}{c}-14 \\
7+6\end{array}$ & $5.3 / 8$ & $2.2^{-0.3}$ & $2.0 \pm 0.3$ & $12^{+4}$ & $9 \pm 3$ & $1.4 \pm 0.2$ & 79685 & $65 \pm 20$ \\
\hline & JEM-X & 2007 Mar 19, 01:15:03 & $1.7^{+0.6}$ & $15^{-7}$ & $3.6 / 13$ & $3.9^{+0.7}$ & $3.6 \pm 0.4$ & $\begin{array}{l}-3 \\
10^{+3}\end{array}$ & $11 \pm 2$ & $1.4 \pm 0.2$ & 212715 & $35 \pm 8$ \\
\hline & XMM & 2007 Mar 31, 01:15:15 & $1.9_{-04}^{-0.3}$ & $7_{-2}^{+3}$ & $8.5 / 9$ & $1.1_{-0.2}^{-2.5}$ & $2.5 \pm 0.3$ & $19_{-2}^{+3}$ & $21 \pm 5$ & $0.6 \pm 0.1$ & 1036812 & $54 \pm 17$ \\
\hline & XMM & 2007 Apr 02, 02:39:03 & $2.2_{-0.3}^{+0.4}$ & $6_{-2}^{-2}$ & $25.5 / 21$ & $1.3_{-0.3}^{+0.2}$ & $3.6 \pm 0.4$ & $21_{-2}^{+2}$ & $28 \pm 5$ & $0.7 \pm 0.1$ & 177828 & $54 \pm 10$ \\
\hline
\end{tabular}

${ }^{a}$ Number of the burst, and instrument that detected it. $*$ designates the burst that was also detected by ISGRI; ${ }^{b}$ blackbody temperature at the peak of the burst, assuming $N_{\mathrm{H}}=12 \times 10^{22} \mathrm{~cm}^{-2} ;{ }^{c}$ Blackbody radius at the peak assuming a distance of $7 \mathrm{kpc}$ to the source; ${ }^{d}$ unabsorbed bolometric flux $(0.1-100 \mathrm{keV})$ at the peak in units of $10^{-8} \mathrm{erg} \mathrm{s}^{-1} \mathrm{~cm}^{-2}$. ${ }^{e}$ Fluence of the burst in units of $10^{-7} \mathrm{erg} \mathrm{cm}^{-2} ;{ }^{f}$ e-folding decay time fitted on the light curve; ${ }^{g}$ e-folding decay time calculated with $\tau_{\text {calc }} \equiv f_{\mathrm{b}} / F_{\text {peak }} ;{ }^{h}$ unabsorbed bolometric persistent flux $(0.1-100 \mathrm{keV})$ prior to the burst in units of $10^{-9} \mathrm{erg} \mathrm{s}^{-1} \mathrm{~cm}^{-2} ;{ }^{i}$ time ellapsed since last detected burst. ${ }^{j}$ Ratio of persistent flux to burst flux: $\gamma \equiv F_{\text {pers }} / F_{\text {peak }}$.

luminosity of GRS 1741.9-2853 corresponds to the boundary of the first two cases, but as we argued above, the energetics of the bursts suggest a pure He explosion, i.e. case (ii), which is strenghtened by the observed rise times and total durations being short.

As a consistency check, we discuss the hydrogen depletion condition. Presuming that $\mathrm{H}$ burns stably on the surface of the neutron star, i.e. case (ii) or (iii), at a rate fixed by $\beta$-decays $\epsilon_{\mathrm{H}}=5.8 \times 10^{15} \times Z_{\mathrm{CNO}} \mathrm{erg} \mathrm{s}^{-1} \mathrm{~g}^{-1}$ (Hoyle \& Fowler 1965) where $Z_{\mathrm{CNO}}$ is the $\mathrm{CNO}$ mass fraction, there is a critical column depth, $y_{\mathrm{d}}$, above which $\mathrm{H}$ is completely depleted leaving only He for the explosion. $y_{\mathrm{d}}$ thus fixes the boundary between cases (ii) and (iii). In the steady-state, $\mathrm{H}$ burns stably as fast as it is accreted, giving $y_{\mathrm{d}}=X_{0} \dot{m} E_{\mathrm{H}} / \epsilon_{\mathrm{H}}$ (Cumming \& Bildsten 2000) where $E_{\mathrm{H}} \approx 6.0 \times 10^{18} \mathrm{erg} \mathrm{g}^{-1}$ is the energy release in the hot $\mathrm{CNO}$ cycle including neutrino energy losses (Wallace \& Woosley 1981). We consider the most intense burst, \#4, which has $y_{\text {ign }} \approx 4.8 \times 10^{8} \mathrm{~g} \mathrm{~cm}^{-2}$. Even in the most extreme case of pure $\mathrm{H}$ accretion $\left(X_{0}=1\right)$, we always find $y_{\text {ign }}>y_{\mathrm{d}}$ as long as $Z_{\mathrm{CNO}}>0.004$. Since a CNO abundance of 0.004 for the companion star is relatively low for a population II low-mass star, we are confident that this condition is fulfilled by the metallicity and thereby that burst 4 was a pure He burst (case (ii)). The conclusion of the comparison between the depletion and ignition columns is not as straightforward as for the other bursts, however, because the assumed values of $X_{0}$ and $Z_{\mathrm{CNO}}$ turn out to be more critical. We note, yet, that GRS 1741.9-2853 is possibly in the central molecular zone of the GC, where Najarro et al. (2009) measured the CNO composition to be up to twice solar, $Z_{\mathrm{CNO}}=0.02$ (see Asplund et al. 2005, for solar abundances) . Provided the donor star is an evolved low-mass star with a Herich composition $\left(X_{0}<0.3\right)$, we again find $y_{\text {ign }}>y_{\mathrm{d}}$ for all the bursts. Because of the large uncertainties in the true values of $X_{0}$ and $Z_{\mathrm{CNO}}$, we emphasize that this depletion condition that relates to case (ii) should be regarded as indicative.

Kuulkers et al. (2008) considered bursts during the quiescent state of a soft X-ray transient (SXT) and the possible trigger role they may play for the SXT outburst itself. For GRS 1741.92853, the burst seen by Swift/BAT in January 2007 would be a good candidate for a trigger of the 2007 outburst. However, the detection of a short activity period, prior to the long 2007 one, in September 2006 (Degenaar \& Wijnands 2009) implies that the companion star was already channelling a substantial amount of matter towards the neutron star at the time. In all likelihood, this helped the SXT outburst develop and then paved the way for type-I bursts, and not the opposite. In addition to this, it is implausible that the source produces bursts during its quiescent state at $10^{32-33} \mathrm{erg} \mathrm{s}^{-1}$. This is theoretically motivated by the expected stability of hydrogen burning via the $p p$-process or pycnonuclear reactions below $10^{33} \mathrm{erg} \mathrm{s}^{-1}$ (Fushiki \& Lamb 1987). This is also observationally corroborated by the non-detection of type-I bursts at this luminosity level until now (Table 1). We note that we explored $2.8 \mathrm{Ms}$ of quiescent state with INTEGRAL over four years without success. At $10^{33} \mathrm{erg} \mathrm{s}^{-1}$, it would also take five years to accumulate the necessary fuel on the neutron star surface for a burst with a characteristic energy release $\sim 10^{39} \mathrm{erg}$. So it looks as if GRS 1741.9-2853 gives rise to thermonuclear explosions only when accreting at a luminosity level in excess of $10^{35-36} \mathrm{erg} \mathrm{s}^{-1}$.

While "burst-only sources" have often been proposed as prototypes of the poorly observed H-burning bursts at low accretion rates, i.e. case (i), and potentially during quiescence, it seems from this discussion that GRS 1741.9-2853 is a member of the faint transient class exhibiting pure helium runaways, i.e. case (ii), only when in outburst. This is another indication that the "burst-only sources" is perhaps not a class on its own. Cornelisse et al. (2004), indeed, already highlighted that the burst-only sources do not form a separate homogeneous group, based on differences in the bursts durations and the nature of the systems (faint transient or quiescent neutron star transients).

\section{Conclusions}

Since its discovery, GRS 1741.9-2853 has not been an easy target for X-ray missions, because it is transient, faint, and located in a region of sky affected by source confusion. As a consequence, the detailed analysis of its bursting behavior needs long exposures, high resolution, and sensitivity, which could finally 
be achieved with INTEGRAL, XMM-Newton, and Swift. In this paper, we have analyzed all the data on GRS 1741.9-2853 ever recorded by the first two satellites since 2002 . We confirm the results of analyses of data acquired by other instruments earlier, i.e., that the source is at a distance of $\sim 7 \mathrm{kpc}$ and exhibits bursts only when in outburst, every couple of years. From the discovery of 15 new bursts in 2005/2007 and the spectral fitting of the contemporaneous persistent emission, we were able to investigate the nuclear burning processes powering the explosions. We find that unstable pure He fusion matches the observations well, whereas the presence of $\mathrm{H}$ would contradict the measured recurrence times and burst durations. In addition, burst oscillations have been proposed to be present in some bursts of GRS 1741.9-2853 by Galloway et al. (2008), which is in line with these oscillations appearing mostly in helium burning runaways (Cumming \& Bildsten 2000; Narayan \& Cooper 2007). However, the existence of a rapidely spinning neutron star in GRS 1741.9-2853 still needs to be conclusively demonstrated by future work with current X-ray facilities.

Acknowledgements. G.T. wishes to warmly thank M. Renaud, J. Chenevez, D. Götz, F. Mattana, G. Ponti, and J.A. Zurita Heras for valuable discussions and help regarding several aspects of the INTEGRAL and XMM-Newton data analysis. E.B. is grateful to M. Perri for his help with the Swift data analysis. M.F. acknowledges the French Space Agency (CNES) for financial support. Part of this work has been funded by the french Agence Nationale pour la Recherche (ANR) through grant ANR-06-JC-0047.

INTEGRAL is an ESA project with instruments and science data center funded by ESA member states (especially the PI countries: Denmark, France, Germany, Italy, Switzerland, and Spain), the Czech Republic, and Poland, and with the participation of Russia and the US. The XMM-Newton project is an ESA Science Mission with instruments and contributions directly funded by ESA Member States and the USA (NASA).

\section{References}

Arnaud, K. A. 1996, in Astronomical Data Analysis Software and Systems V, ed. G. H. Jacoby, \& J. Barnes, ASP Conf. Ser., 101,

Asplund, M., Grevesse, N., \& Sauval, A. J. 2005, in Cosmic Abundances as Records of Stellar Evolution and Nucleosynthesis, ed. T. G. Barnes, III, \& F. N. Bash, ASP Conf. Ser., 336, 25

Bildsten, L. 1998, in NATO ASIC Proc. 515: The Many Faces of Neutron Stars, ed. R. Buccheri, J. van Paradijs, \& A. Alpar, 419

Brandt, S., Budtz-Jørgensen, C., Lund, N., et al. 2003, A\&A, 411, L243

Chelovekov, I. V., Grebenev, S. A., \& Sunyaev, R. A. 2007, [arXiv:0709.2328]

Cocchi, M., Bazzano, A., Natalucci, L., et al. 1999, A\&A, 346, L45

Cocchi, M., Bazzano, A., Natalucci, L., et al. 2001, A\&A, 378, L37

Cooper, R. L., \& Narayan, R. 2007, ApJ, 661, 468

Cornelisse, R., in't Zand, J. J. M., Verbunt, F., et al. 2003, A\&A, 405, 1033

Cornelisse, R., in't Zand, J. J. M., Kuulkers, E., et al. 2004, Nucl. Phys. B Proc. Suppl., 132, 518

Courvoisier, T. J.-L., Walter, R., Beckmann, V., et al. 2003, A\&A, 411, L53

Cumming, A., \& Bildsten, L. 2000, ApJ, 544, 453

Degenaar, N., \& Wijnands, R. 2009, A\&A, 495, 547

Dodds-Eden, K., Porquet, D., Trap, G., et al. 2009 [arXiv: 0903. 3416]

Falanga, M. 2008, in Amer. Instit. Phys. Conf. Ser., 1054, 157

Falanga, M., Chenevez, J., Cumming, A., et al. 2008, A\&A, 484, 43

Falanga, M., Cumming, A., Bozzo, E., et al. 2009, A\&A, 496, 333

Fox, D. B., Barthelmy, S. D., \& Markwardt, C. B. 2007, GCN Circ., 6020

Fujimoto, M. Y., Hanawa, T., \& Miyaji, S. 1981, ApJ, 247, 267

Fujimoto, M. Y., Sztajno, M., Lewin, W. H. G., et al. 1987, ApJ, 319, 902
Fushiki, I., \& Lamb, D. Q. 1987, ApJ, 323, L55

Galloway, D. K., Muno, M. P., Hartman, J. M., Psaltis, D., \& Chakrabarty, D. 2008, ApJS, 179, 360

Gehrels, N., Chincarini, G., Giommi, P., et al. 2004, ApJ, 611, 1005

Goldwurm, A. 1996, Mem. Soc. Astron. Ital., 67, 121

Goldwurm, A., David, P., Foschini, L., et al. 2003, A\&A, 411, L223

Heise, J., in't Zand, J. J. M., Smith, M. J. S., et al. 1999, Astrophys. Lett. Comm., 38, 297

Hoyle, R., \& Fowler, W. A. 1965, in Quasi-Stellar Sources and Gravitational Collapse, ed. I. Robinson, A. Schild, \& E. L. Shucking (Chicago Univ. Press), 17

in't Zand, J. 2001, in Exploring the Gamma-Ray Universe, ed. A. Gimenez, V. Reglero, \& C. Winkler, ESA SP-459, 463

Jansen, F., Lumb, D., Altieri, B., et al. 2001, A\&A, 365, L1

King, A. R. 2000, MNRAS, 315, L33

King, A. R., \& Ritter, H. 1998, MNRAS, 293, L42

Kuulkers, E., den Hartog, P. R., in't Zand, J. J. M., et al. 2003, A\&A, 399, 663

Kuulkers, E., Shaw, S., Chenevez, J., et al. 2007a, The Astronomer's Telegram, 1005,1

Kuulkers, E., Shaw, S., Chenevez, J., et al. 2007b, The Astronomer's Telegram, 1008,1

Kuulkers, E., Shaw, S. E., Paizis, A., et al. 2007c, A\&A, 466, 595

Kuulkers, E., in 't Zand, J. J. M., \& Lasota, J. P. 2008, [arXiv: 0809. 3323]

Lasota, J.-P. 2001, New Astron. Rev., 45, 449

Laycock, S., Zhao, P., Torres, M. A. P., et al. 2005, The Astronomer's Telegram, 522,1

Lebrun, F., Leray, J. P., Lavocat, P., et al. 2003, A\&A, 411, L141

Lewin, W. H. G., van Paradijs, J., \& Taam, R. E. 1993, Space Sci. Rev., 62, 223

Liu, Q. Z., van Paradijs, J., \& van den Heuvel, E. P. J. 2007, A\&A, 469, 807

Lund, N., Budtz-Jørgensen, C., Westergaard, N. J., et al. 2003, A\&A, 411, L231

Mereghetti, S., Götz, D., Borkowski, J., Walter, R., \& Pedersen, H. 2003, A\&A, 411, L291

Muno, M., Baganoff, F., \& Arabadjis, J. 2003, ApJ, 598, 474

Muno, M. P., Bauer, F. E., Bandyopadhyay, R. M., \& Wang, Q. D. 2006, ApJS, 165,173

Muno, M. P., Wijnands, R., Wang, Q. D., et al. 2007, The Astronomer's Telegram, 1013, 1

Najarro, F., Figer, D. F., Hillier, D. J., Geballe, T. R., \& Kudritzki, R. P. 2009, ApJ, 691, 1816

Narayan, R., \& Cooper, R. L. 2007, ApJ, 665, 628

Peng, F., Brown, E. F., \& Truran, J. W. 2007, ApJ, 654, 1022

Porquet, D., Grosso, N., Goldwurm, A., et al. 2007, The Astronomer's Telegram, 1058, 1

Porquet, D., Grosso, N., Predehl, P., et al. 2008, A\&A, 488, 549

Sakano, M., Koyama, K., Murakami, H., Maeda, Y., \& Yamauchi, S. 2002, ApJS, 138,19

Strohmayer, T., \& Bildsten, L. 2006, New views of thermonuclear bursts (Compact stellar X-ray sources), 113

Strohmayer, T. E., Jahoda, K., Giles, A. B., et al. 1997, ApJ, 486, 355

Sunyaev, R. 1990, IAU Circ., 5104, 1

Sunyaev, R., Pavlinskii, M., Churazov, M. G. E., et al. 1991, Astron. Lett., 17, 42

Trap, G., Goldwurm, A., Terrier, R., et al. 2009, in Proceedings of the 7 th INTEGRAL Workshop, An INTEGRAL View of Compact Objects, PoS(Integral108)047

Ubertini, P., Lebrun, F., Di Cocco, G., et al. 2003, A\&A, 411, L131

Wallace, R. K., \& Woosley, S. E. 1981, ApJS, 45, 389

Westergaard, N. J., Kretschmar, P., Oxborrow, C. A., et al. 2003, A\&A, 411, L257

Wijnands, R. 2006, in Bull. Amer. Astron. Soc., 38, 1183

Wijnands, R. 2008, Proceedings of A decade of accreting millisecond pulsars

Wijnands, R., Maccarone, T., Miller-Jones, J., et al. 2005, The Astronomer's Telegram, 512, 1

Wijnands, R., in't Zand, J. J. M., Rupen, M., et al. 2006, A\&A, 449, 1117

Wijnands, R., Klien Wolt, M., Kuulkers, E., et al. 2007, The Astronomer's Telegram, 1006, 1

Winkler, C., Courvoisier, T. J.-L., Di Cocco, G., et al. 2003, A\&A, 411, L1 